\title{
Color-Critical Graphs and Hypergraphs With FeW Edges: A Survey
}

\section{A. KOSTOCHKA*}

The current situation with bounds on the smallest number of edges in colorcritical graphs and hypergraphs is discussed.

\section{INTRODUCTION}

The theory of graph and hypergraph coloring plays a central role in discrete mathematics. It has applications in areas with seemingly little connection to coloring. Coloring deals with the fundamental problem of partitioning a set of objects into classes that avoid certain conflicts. Many timetabling, sequencing, and scheduling problems are of this nature.

A hypergraph is color-critical if deleting any edge or vertex reduces the chromatic number; a color-critical hypergraph with chromatic number $k$ is $k$-critical. Every $k$-chromatic hypergraph contains a $k$-critical hypergraph, so one can study chromatic number by studying the structure of $k$-critical (hyper)graphs. There is vast literature on $k$-critical graphs and hypergraphs. Many references can be found in [23, Chapters 5 and 1].

In this survey we concentrate on $k$-critical graphs and hypergraphs with few edges. Lower bounds on, say, average degree of $k$-critical graphs can be applied as follows. If we know that the average degree of every $k$ critical graph in a family $\mathcal{H}$ is at least $x$, and the average degree of every subgraph $H^{\prime}$ of a graph $H \in \mathcal{H}$ is less than $x$, then we know that $H$ is $(k-1)$-colorable. For example, a theorem of Gallai (described in Section 4

\footnotetext{
${ }^{*}$ This work was partially supported by the NSF grant DMS-0099608.
} 
below) says that $|E(G)| /|V(G)| \geq 0.5\left(k-1+\frac{k-3}{k^{2}-3}\right)$ when $G$ is a $k$ critical graph other than $K_{k}$. For $k \geq 6$ and arbitrary $g$, this implies that the problem of testing $k$-colorability is solvable in polynomial time for graphs that embed on the orientable surface of genus $g$. If such a graph is not $k$-colorable, then it has a $(k+1)$-critical subgraph $G^{\prime}$. Euler's Formula yields $\left|E\left(G^{\prime}\right)\right| \leq 3\left(\left|V\left(G^{\prime}\right)\right|+2 g-2\right)$, but Gallai's Theorem requires $\left|E\left(G^{\prime}\right)\right| \geq 0.5\left(k+\frac{k-2}{k^{2}+2 k-2}\right)\left|V\left(G^{\prime}\right)\right|$. For $k \geq 6$, this requires $\left|V\left(G^{\prime}\right)\right| \leq 138(g-1)$. Therefore, it suffices to test the $k$-colorability of every subgraph of $G$ having at most $138(g-1)$ vertices.

Another application of such bounds to coloring of graphs on surfaces appears in [7], and Krivelevich [36] presents interesting applications to random graphs.

In connection with list coloring originated by Vizing [54] and Erdös, Rubin, and Taylor [19], one can study also list-k-critical (hyper)graphs. Given a (hyper)graph $G$ and a list assignment $L$ for the vertices of $G, G$ is $L$-critical if there is no proper coloring of vertices of $G$ from their lists, but after deleting any edge or vertex, such a coloring does exist. A list assignment $L$ for the vertices of a (hyper)graph $G$ is called $t$-uniform if $|L(v)|=t$ for every $v \in V(G)$.

Two basic questions will be discussed:

(a) what is the minimum possible number of edges in a $k$-critical (hyper)graph in a given class $\mathcal{G}$ ?

(b) what is the minimum possible number of edges in a $k$-critical (hyper)graph on $n$ vertices in a given class $\mathcal{G}$ ? In particular what is the inf $\frac{|E(G)|}{|V(G)|}$ taken over $k$-critical (hyper)graph in a given class $\mathcal{G}$ ?

In the next section we give proofs of a few basic facts. Then graph questions are discussed in Sections 3, 4, and 5, and hypergraph questions in Sections 6, 7, and 8 .

\section{Preliminaries}

It is well known that color-critical graphs and hypergraphs do not have vertices of small degree. This is true also for list-critical hypergraphs. We state this folklore observation as a proposition because of its importance. 
Proposition 1. Let $L$ be a $(k-1)$-uniform list for a hypergraph $G$ and let $G$ be $L$-critical. Then $\operatorname{deg}_{G}(v) \geq k-1$ for every $v \in V(G)$. Moreover, for every $v \in V(G)$, there exist some $k-1$ edges $e_{1}, \ldots, e_{k-1}$ such that $e_{i} \cap e_{j}=\{v\}$ for every $1 \leq i<j \leq k-1$.

Proof. Let $v \in V(G)$. By definition, there is an $L$-coloring $f$ of $G-v$, but we cannot extend $f$ to $v$. This means that for every $\alpha \in L(v)$, there exists an edge $e_{\alpha}$ containing $v$ such that all vertices of $e_{\alpha}-v$ are colored with $\alpha$. And for distinct $\alpha, \beta \in L(v)$, the sets $e_{\alpha}-v$ and $e_{\beta}-v$ must be disjoint. This proves the proposition.

Dirac [12] observed that $k$-critical hypergraphs have not only the minimum degree at least $k-1$, but also the edge-connectivity at least $k-1$.

Proposition 2 [12]. Let $G$ be a $k$-critical hypergraph. Then $G$ is $(k-1)$ edge-connected.

Proof. ${ }^{1}$ Assume that $V(G)=W \cup U, W \cap U=\emptyset$, and the only edges intersecting both $W$ and $U$ are $e_{1}, \ldots, e_{s}$, where $s \leq k-2$. For $j=1, \ldots, s$, let $w_{j}$ be some vertex in $W \cap e_{j}$ and $u_{j}$ be some vertex in $U \cap e_{j}$. Since $G$ is $k$-critical, there exists a $(k-1)$-coloring $f_{W}$ of $G(W)$ and a $(k-1)$-coloring $f_{U}$ of $G(U)$, both using colors $1, \ldots, k-1$. We can change the names of colors in $f_{U}$ in $(k-1)$ ! ways, keeping the same partition of vertices. For a given $j$, in at most $(k-2)$ ! ways we will get the colors of $w_{j}$ and $u_{j}$ the same. Thus, there are at least $(k-1) !-(k-2) ! s=(k-1-s)(k-2) !>0$ ways to choose the names of colors in $f_{U}$ so that the resulting $(k-1)$-coloring of $G$ will be proper.

In view of the simple proof of Proposition 2 above, it is a bit surprising that for list colorings, this proposition does not hold.

Example 1. Let $H(k)$ denote the graph with $V(H(k))=W \cup U$, where $W=\left\{w_{1}, \ldots, w_{k}\right\}, U=\left\{u_{1}, \ldots, u_{k}\right\}$, such that the subgraphs of $H(k)$ induced by $W$ and $U$ are complete graphs and there is exactly one edge, namely $w_{k} u_{k}$, connecting $W$ with $U$.

Define the list $L$ for the $H(k)$ by

$$
L(v)= \begin{cases}\{1, \ldots, k-1\}, & \text { if } v \in V(G)-\left\{w_{k}, u_{k}\right\} \\ \{2, \ldots, k\}, & \text { if } v \in\left\{w_{k}, u_{k}\right\}\end{cases}
$$

\footnotetext{
${ }^{1}$ I've learned this proof from Jacent Tokaz via Douglas West. Another short proof the reader can find in [52].
} 
Assume that $H(k)$ is $L$-colorable. Then all colors $1, \ldots, k-1$ should be used on $w_{1}, \ldots, w_{k-1}$, and the same holds for $u_{1}, \ldots, u_{k-1}$. Thus, both $w_{k}$ and $u_{k}$ must be colored with $k$, a contradiction. It is also easy to check that after deleting any edge, we get an $L$-colorable graph. Hence, $H(k)$ is $L$-critical and has connectivity 1 .

\section{DiRAC-TYPE BOUNDS}

Critical graphs were first defined and used by Dirac [11] in 1951. Dirac was interested in

$$
F(k, n)=\min \{|E(G)|: G \text { is } k \text {-critical and }|V(G)|=n\} \text {. }
$$

In view of Proposition 1 , for every $k$-critical graph $G$ on $n$ vertices,

$$
\sum_{v \in V(G)} \operatorname{deg}(v) \geq(k-1) n
$$

Thus $2 F(k, n) \geq(k-1) n$. This motivates introducing the excess

$$
\varepsilon(k, G)=\sum_{v \in V(G)}(\operatorname{deg}(v)-k+1)
$$

and

$$
\begin{aligned}
\varepsilon(k, n) & =\min \{\varepsilon(k, G) \mid G \text { is } k \text {-critical and }|V(G)|=n\} \\
& =2 F(k, n)-(k-1) n .
\end{aligned}
$$

Brooks' Theorem yields that $\varepsilon(k, n) \geq 1$ for $k \geq 4$ and $n \geq k+1$. Dirac [13] proved the following.

Theorem 3 [13]. Let $k \geq 4$ and $G$ be a $k$-critical graph. If $G$ is not a $K_{k}$, then $\varepsilon(k, G) \geq k-3$.

Shorter and more elegant proofs of this result were given by Kronk and Mitchem [37] and Weinstein [56]. We present here a proof using ideas from $[10,29]$. 
Proof of Dirac's Theorem. Assume that $G$ is a vertex minimum $k$-critical graph distinct from $K_{k}$, with $\varepsilon(k, G) \leq k-4$. For every $v \in V(G)$, define $\varepsilon_{G}(k, v)=\operatorname{deg}_{G}(v)-k+1$. Then $\varepsilon(k, G)=\sum_{v \in V(G)} \varepsilon_{G}(k, v)$.

Let $w \in V(G)$ and $\operatorname{deg}_{G}(v)=k-1$. Since $G \neq K_{k}$, there are nonadjacent vertices $x_{1}, x_{2} \in N_{G}(w)$. The graph $G^{*}$ obtained from $G$ by merging $x_{1}$ and $x_{2}$ into a new vertex $x^{*}$ is not $(k-1)$-colorable, since every its $(k-1)$-coloring generates a $(k-1)$-coloring of $G$. Hence $G^{*}$ contains a $k$-critical subgraph $G_{1}^{*}$. Note that $x^{*} \in V\left(G_{1}^{*}\right)$ (otherwise, $G_{1}^{*}$ would be a subgraph of $G)$. Since $\operatorname{deg}_{G^{*}}(w)=k-2, w \neq V\left(G_{1}^{*}\right)$.

Let $V_{1}^{*}=V\left(G_{1}^{*}\right), V_{1}=V_{1}^{*}-x^{*}+x_{1}+x_{2}$, and $V_{2}=V\left(G^{*}\right)-V_{1}^{*}=$ $V(G)-V_{1}$. Let $E_{1,2}$ be the set of edges connecting $V_{1}$ with $V_{2}$ in $G$. Assume that $G_{1} \neq K_{k}$. Then by the minimality of $G, \varepsilon\left(k, G_{1}\right) \geq k-3$. Since every edge in $E_{1,2}$ contributes 1 to $\sum_{v \in V_{1}^{*}}\left(\varepsilon_{G^{*}}(k, v)-\varepsilon_{G_{1}^{*}}(k, v)\right)$, we have

$$
\varepsilon(k, G) \geq \sum_{v \in V_{2}} \varepsilon_{G}(k, v)+\varepsilon\left(k, G_{1}^{*}\right)+\left|E_{1,2}\right|-(k-1),
$$

where the last $-(k-1)$ reflects merging $x_{1}$ with $x_{2}$. By Proposition 2, $\left|E_{1,2}\right| \geq k-1$. Hence (2) yields $\varepsilon(k, G) \geq k-3$, a contradiction.

Thus $G^{*}$ contains a $K_{k}$ one of whose vertices is $x^{*}$. In other words, $G$ contains a triple $\left(M, y_{1}, y_{2}\right)$, where $M$ is a clique of size $k-1$ and $y_{1}$ and $y_{2}$ are non-adjacent vertices with $N_{G}\left(\left\{y_{1}, y_{2}\right\}\right) \supset M$.

Among all such triples, choose a triple $\left(M, y_{1}, y_{2}\right)$ with maximum $\left|N_{G}\left(y_{1}\right) \cap M\right|$.

Now, let $x_{1}=y_{1}$ if $N_{G}\left(y_{1}\right) \cap M$ contains a vertex $w$ of degree $k-1$, and let $x_{1}=y_{2}$ otherwise. Since $\varepsilon(k, G) \leq k-4$, in both cases there is $w \in N_{G}\left(x_{1}\right) \cap M$ with $\operatorname{deg}_{G}(w)=k-1$. Let $x_{2}$ be a non-adjacent to $x_{1}$ vertex in $M$ of the smallest degree. Define graphs $G^{*}$ and $G_{1}^{*}$ and sets $V_{1}^{*}$, $V_{1}, V_{2}$, and $E_{1,2}$ as above. Then again $G_{1}^{*}=K_{k}$. Let $M^{\prime}=V\left(G_{1}^{*}\right)-x^{*}$, $M^{*}=M-x_{2}+x^{*}, M_{1}^{*}=M^{*} \cap V\left(G_{1}^{*}\right)$ and $m_{1}=\left|M_{1}^{*}\right|$. Since every $v \in M_{1}^{*}$ has at least $k-1$ neighbors in $G_{1}^{*}$ and at least $k-1-m_{1}$ neighbors outside of $G_{1}^{*}, \varepsilon(k, G) \geq\left(m_{1}-1\right)\left(k-1-m_{1}\right)$. Since $w \notin M_{1}^{*}$, in order to have $\left(m_{1}-1\right)\left(k-1-m_{1}\right) \leq k-4$, we need $m_{1}=1$, which means $M_{1}^{*}=\left\{x^{*}\right\}$, i.e. $M^{\prime} \cap M^{*}=\emptyset$.

Let $\left|N_{G}\left(y_{1}\right) \cap M\right|=m$ and $\left|N_{G}\left(x_{1}\right) \cap M\right|=m^{\prime}$. Then $\left|N_{G}\left(x_{2}\right) \cap M\right| \geq$ $k-1-m$ by the choice of $y_{1}$. Hence $\operatorname{deg}_{G}\left(x_{2}\right) \geq(k-2)+(k-1-m)$. By the choice of $x_{2}, \varepsilon_{G}(k, v) \geq k-2-m$ for every $v \in M-N_{G}\left(x_{1}\right)$. Taking 
into account that all vertices in $M^{\prime} \cup M-x_{2}$ are adjacent to $x_{1}$ or $x_{2}$, and at least $m^{\prime}$ of them to both, we have

$$
\varepsilon(k, G) \geq(k-2-m)\left(k-2-m^{\prime}\right)+m^{\prime}-1 .
$$

If $x_{1}=y_{1}$, then $m^{\prime}=m$ and the minimum of $(k-2-m)^{2}+m-1$ over integers $m, 1 \leq m \leq k-2$ is exactly $k-3$. This contradicts the choice of $G$. So, let $x_{1}=y_{2}$. In this case, $y_{1}$ is not adjacent to at least 3 vertices of degree $k-1$ in $M$, and hence $k-2-m \geq 2$. Then (3) yields $\varepsilon(k, G) \geq 2\left(k-2-m^{\prime}\right)+m^{\prime}-1 \geq k-3$, again. This proves the theorem.

For $k \geq 3$, let $\mathcal{D}_{k}$ denote the family of all graphs $G$ whose vertex set consists of three non-empty pairwise disjoint sets $A, B_{1}, B_{2}$ with $\left|B_{1}\right|+$ $\left|B_{2}\right|=|A|+1=k-1$ and two additional vertices $a, b$ such that $A$ and $B_{1} \cup B_{2}$ are cliques in $G$ not joined by any edge, $N_{G}(a)=A \cup B_{1}$ and $N_{G}(b)=A \cup B_{2}$. Obviously, such a graph $G$ has $2 k-1$ vertices, $\operatorname{deg}_{G}(x)=k-1$ for all vertices $x \neq a, b$, and $\varepsilon(k, G)=\operatorname{deg}_{G}(a)+\operatorname{deg}_{G}(b)-2(k-1)=k-3$. That $G$ is $k$-critical was observed by Dirac [13] and by Gallai [20]. Thus Dirac's bound is sharp for every $G \in \mathcal{D}_{k}$.

In 1974, Dirac [14] extended Theorem 3 as follows.

Theorem 4 [14]. Let $k \geq 4$, and let $G$ be a $k$-critical graph. If $G$ is neither the $K_{k}$ nor a member of $\mathcal{D}_{k}$, then

$$
\varepsilon(k, G) \geq \begin{cases}2 & \text { if } k=4, \\ k-1 & \text { if } k \geq 5 .\end{cases}
$$

Shorter proofs of this result were found by Mitchem [40] and by Deuber et. al. [10].

For $k \geq 3$, let $\mathcal{F}_{k}$ denote the family of all graphs $G$ whose vertex set consists of four non-empty pairwise disjoint sets $A_{1}, A_{2}, B_{1}, B_{2}$, where $\left|B_{1}\right|+\left|B_{2}\right|=\left|A_{1}\right|+\left|A_{2}\right|=k-1$ and $\left|A_{2}\right|+\left|B_{2}\right| \leq k-1$, and one additional vertex $c$ such that $A=A_{1} \cup A_{2}$ and $B=B_{1} \cup B_{2}$ are cliques in $G$, $N_{G}(c)=A_{1} \cup B_{1}$, and a vertex $a \in A$ is joined to a vertex $b \in B$ by an edge in $G$ if and only if $a \in A_{2}$ and $b \in B_{2}$. Every such graph $G$ has $2 k-1$ vertices and independence number 2 . Consequently, $G$ is not $(k-1)$ colorable. Moreover, it is easy to check that the deletion of any edge results in a $(k-1)$-colorable graph. Therefore, $G$ is $k$-critical. Clearly, $G$ is in $\mathcal{D}_{k}$ if and only if $\left|A_{2}\right|=1$ or $\left|B_{2}\right|=1$. Moreover, $\mathcal{D}_{k} \subseteq \mathcal{F}_{k}$. 
Kostochka and Stiebitz [29] improved the bounds of Theorem 4 as follows.

Theorem 5 [29]. Let $k \geq 4$ and $G$ be a $k$-critical graph. If $G$ is neither a $K_{k}$ nor a member of $\mathcal{F}_{k}$, then $\varepsilon(k, G) \geq 2(k-3)$.

The bounds of this result are tight not only for graphs on $2 k-1$ vertices. There are examples of $k$-critical graphs $G$ with $\varepsilon(k, G)=2(k-3)$ on $k+2,2 k-2,2 k-1,2 k$ and $3 k-2$ vertices. However, for $k \geq 4$, it is possible to show that $\varepsilon(k, n)=2(k-3)$ if and only if $n \in\{k+2,2 k-2,2 k, 3 k-2\}$.

The join of vertex disjoint graphs $G_{1}$ and $G_{2}$, denoted by $G_{1} \vee G_{2}$, is the graph obtained from their union by adding edges joining each vertex of $G_{1}$ to each vertex of $G_{2}$. It is evident that $\chi\left(G_{1} \vee G_{2}\right)=\chi\left(G_{1}\right)+\chi\left(G_{2}\right)$. Moreover, $G_{1} \vee G_{2}$ is critical if and only if both $G_{1}$ and $G_{2}$ are critical.

In one of his seminal papers from 1963, Gallai [21] proved that every $k$-critical graph with at most $2 k-2$ vertices is the join of two other critical graphs. This allowed him to find the minimum excess of $k$-critical graphs with at most $2 k-1$ vertices and to describe the extremal cases.

Theorem 6 [21]. Let $k, p$ be integers satisfying $k \geq 4$ and $2 \leq p \leq k-1$. If $G$ is a $k$-critical graph with $k+p$ vertices, then $\varepsilon(k, G) \geq p(k-p)-2$, where equality holds if and only if $G$ is the join of $K_{k-p-1}$ and a graph in $\mathcal{D}_{p+1}$.

Since Proposition 1 holds for list coloring, one might expect that for every $(k-1)$-uniform list $L$, each $L$-critical graph $G$ on $n>k$ vertices has $\varepsilon(k, G) \geq k-3$. But Example 1 shows an $L$-critical graph $H(k)$ with $\varepsilon(k, H(k))=2$ for every $k$. On the other hand, if we forbid $K_{k}$ as a subgraph, the situation changes.

Theorem 7 [31]. Let $k \geq 4$. Let $G$ be a hypergraph on $n$ vertices not containing $K_{k}$, and let $L$ be a list for $G$ with $|L(v)|=k-1$ for every $v \in V(G)$. If $G$ is $L$-critical, then $2|E(G)| \geq(k-1) n+k-3$. In particular, if $G$ is a graph, then $\varepsilon(k, G) \geq k-3$.

The above results determine the values of $\varepsilon(k, n)$ (and hence $F(k, n)$ ) for $n \leq 2 k$ and $n=3 k-2$. Hajós construction with one of the graphs being $K_{k}$ yields that

$$
\varepsilon(k, n+k-1) \leq \varepsilon(k, n)+k-3 .
$$


Ore [42] suggested that (4) holds with equality for every $n \geq k+2$ (see also [23, p. 99]). In view of the above results, that would mean that

$$
\lim _{n \rightarrow \infty} \frac{2 F(k, n)}{n}=k-\frac{2}{k-1} .
$$

The existing lower bounds are far from (5). The next section contains more discussion on the topic.

\section{Gallai-TyPe BOUnds}

The results of the previous section give bounds on $\varepsilon(k, n)$ that do not depend on $n$, while (5) (if true) would imply that $\varepsilon(k, n)$ grows asymptotically as $n(k-3) /(k-1)$. There is an attractive conjecture that for $n \geq 6$,

$$
F(4, n) \geq\left\lfloor\frac{5 n}{3}\right\rfloor
$$

The first lower bound on $\varepsilon(k, n)$ depending on $n$ was the abovementioned theorem of Gallai [20].

Theorem 8 [20]. Let $G$ be a $k$-critical graph. Then every block in the subgraph of $G$ induced by vertices of degree $k-1$ is a complete graph or an odd cycle. Furthermore, if $k \geq 4$ and $G \neq K_{k}$, then

$$
2|E(G)| \geq\left(k-1+\frac{k-3}{k^{2}-3}\right)|V(G)|
$$

In particular, if $k \geq 4$ and $n \geq k+2$, then

$$
\varepsilon(k, n) \geq \frac{k-3}{k^{2}-3} n .
$$

For $n=2 k$ this gives only $\varepsilon(k, n) \geq 2$ while Theorem 5 gives $\varepsilon(2 n, n) \geq$ $2(k-3)$; but in the long run the bound of Theorem 5 is much better.

Remark 1. The proof of Theorem 8 works for list coloring as well, so Inequality (7) holds also for every $L$-critical graph $G \neq K_{k}$ if $L$ is a $(k-1)$ uniform list for $G$ and $k \geq 4$. 
Theorem 8 yields $F(4, n) \geq\left(\frac{3}{2}+\frac{1}{26}\right) n$ while the conjecture (6) is that $F(4, n)$ is roughly $\left(\frac{3}{2}+\frac{1}{6}\right) n$.

Krivelevich [35, 36], using a result of Stiebitz [49] on the structure of critical graphs, improved this bound as follows.

Theorem 9 [35, 36]. Suppose $k \geq 4$, and let $G$ be a $k$-critical graph on more than $k$ vertices. Then

$$
2|E(G)| \geq\left(k-1+\frac{k-3}{k^{2}-2 k-1}\right)|V(G)| .
$$

In particular, if $k \geq 4$ and $n \geq k+2$, then

$$
\varepsilon(k, n) \geq \frac{k-3}{k^{2}-2 k-1} n .
$$

The improvement is better for small $k$. In particular, it gives $F(4, n) \geq$ $\left(\frac{3}{2}+\frac{1}{14}\right) n$ for $n \geq 6$. Since Stiebitz's result [49] does not hold for list colorings, the proof of Theorem 9 does not generalize to list critical graphs.

Kostochka and Stiebitz [32] improved Krivelevich's bound for $k \geq 9$.

Theorem 10 [32]. Suppose $k \geq 6$, and let $G$ be a $k$-critical graph on more than $k$ vertices. Then

$$
2|E(G)| \geq\left(k-1+\frac{2(k-3)}{k^{2}+6 k-9-\frac{6}{k-2}}\right)|V(G)| .
$$

In particular, if $k \geq 4$ and $n \geq k+2$, then

$$
\varepsilon(k, n) \geq \frac{2(k-3)}{k^{2}+6 k-9-\frac{6}{k-2}} n .
$$

The technique of [32] generalizes to list colorings, with sufficiently weaker bounds.

Theorem 11 [32]. Suppose $k \geq 9$, and let $G$ be an $L$-critical graph, where $L$ is a $(k-1)$-uniform list for $G$. If $G \neq K_{k}$, then

$$
2|E(G)| \geq\left(k-1+\frac{1.2(k-3)}{k^{2}+k-4-\frac{4}{5 k-10}}\right)|V(G)| .
$$

There is still a gap of roughly $\frac{k-4}{k-1} n$ between (12) and the known upper bounds on $\varepsilon(k, n)$. And the conjecture (6) is an attractive challenge. 


\section{Critical graphs With no large Cliques}

It is natural to ask whether the bound on the number of edges in a $k$ critical graph with $n$ vertices can be improved when we have additional restrictions on the structure of the graph. A possible direction is to ask what is $F(k, n, s)$ - the minimum number of edges in a $k$-critical graph on $n$ vertices without cliques of size $s+1$.

Together with Theorem 8, Dirac [13] proved the bound

$$
2 F(k, n, s) \geq(k-1) n+(k-3)+(k-s) \quad \text { if } \quad s \leq k \leq n-2 .
$$

Weinstein [56] improved the bound to $2 F(k, n, s) \geq(k-1) n+(k-3)+$ $2(k-s)$, but the surplus over $(k-1) n$ still does not depend on $n$.

Krivelevich [36] improved the bound as follows.

Theorem 12 [36]. Let $k$ and $s$ be integers satisfying $3 \leq s<k$. Let $G$ be a $k$-critical graph not containing a clique of size $s+1$. Then

1. if $s \leq 2 k / 3$, then $|E(G)| \geq\left(\frac{k}{2}-\frac{k-2}{2(2 k-s-3)}\right)|V(G)|$;

2. if $s \geq 2 k / 3$, then $|E(G)| \geq\left(\frac{k}{2}-\frac{(k-2) s}{2\left(2 k s-2 k-s^{2}\right)}\right)|V(G)|$;

Krivelevich [36] also gives a bit stronger bounds on the number of edges for critical graphs without short odd cycles, and shows nice applications of his bounds to other interesting problems.

The case of fixed $s$ and large $k$ was considered by Kostochka and Stiebitz [30].

Theorem 13 [30]. For every fixed $s$ and sufficiently large $k$, every L-critical graph $G$ on $n$ vertices without cliques of size $s+1$ for any $(k-1)$-uniform list $L$ has at least $(k-o(k)) n$ edges. In particular, $F(k, n, s) \geq(k-o(k)) n$.

This bound is almost twice larger than the previously mentioned bounds for large $k$. The bad side of the theorem is that it works only for really large $k$, when Johannson's theorem on coloring of sparse graphs with given maximum degree works. The good side of it is that the theorem is asymptotically (in $k$ ) tight even for graphs of arbitrary girth. A way to construct $k$-critical graphs $G$ of arbitrary girth with $|E(G)| /|V(G)|<k-1$ was shown in [24]. 
Abbott, Hare, and Zhou [3] constructed $k$-critical graphs $G$ with density $|E(G)| /|V(G)|<k-7 / 3$ for girth 4 and density $|E(G)| /|V(G)|<k-2$ for girth 5. Kostochka and Nešetrril [28] proved that there exist $k$-critical graphs $G$ with $|E(G)| /|V(G)|<k-2$ and arbitrarily large girth.

But for small and moderate $k$, finding least possible average degree of a triangle-free $k$-critical graph is an interesting open problem.

\section{CRitical hypergraphs With FeW EDGES}

Famous Local Lemma [18] implies that every $k$-critical $r$-uniform hypergraph has maximum degree at least $(k-1)^{r-1} / 4 r$. One might expect that the average degree of $k$-critical $r$-uniform hypergraphs is also always superlinear in $k$ for fixed $r$. In fact, Erdős and Lovász [18, p. 612] conjectured this for simple hypergraphs. But this is not the case.

Lovász [38, 39], Woodall [53], Seymour [47], and Burstein [9] proved that $|E(H)| \geq|V(H)|$ for every 3-critical hypergraph $H$. Kostochka and Nešetřil [28] extended results of Burstein [9] and of Abbott, Hare, and Zhou $[1,3]$ by proving the following upper bound on the minimum of $|E(H)|$ in terms of $|V(H)|$.

Theorem 14 [28]. For each $r \geq 3, k \geq 4, g \geq 3$ and $\varepsilon>0$, there exists an $r$-uniform $k$-critical hypergraph $H$ with girth at least $g$ and $|E(H)| /|V(H)|<k-2+\varepsilon$.

And for large $k$, this is almost matched by the following lower bound due to Kostochka and Stiebitz [30].

Theorem 15 [30]. Let $H$ be a hypergraph with no edges of size 2 . If $H$ is $L$-critical for a $k$-uniform list assignment $L$, then $|E(H)| /|V(H)| \geq$ $k(1-3 / \sqrt[3]{k})$.

The advantage of Theorem 15 is that it works for list coloring, and not only for uniform hypergraphs. The girth is also not an issue. The disadvantage is that it provides no information when $k<27$.

Note that all known examples of $r$-uniform $k$-critical hypergraph with small average degree have many vertices. Thus it makes sense to ask about $m(r, k)$ - the minimum number of edges in an $r$-uniform not $k$-colorable hypergraph (with no restriction on the number of vertices). A first thought 
here would be that the complete $r$-uniform hypergraph on $1+k(r-1)$ vertices gives the answer. And for $r=2$ (ordinary graphs) this is the case. But already for $r=3$ the Fano plane with 7 edges beats $K_{5}^{3}$ with 10 edges. Erdös and Hajnal [17] suggested that if $k$ is very large in comparison with $r$, then the complete hypergraph still is the best construction, but Alon [4] disproved this conjecture.

Finding good estimates on $m(r, k)$, and especially on $m(r, 2)$, was one of the favorite topics of Paul Erdős for a long time. He proved in $[15,16]$ the first nontrivial bounds on $m(r, 2)$ :

$$
2^{r-1} \leq m(r, 2) \leq r^{2} 2^{r} .
$$

The proofs of both upper and lower bounds are simple, so we present them here.

Lemma 16. For every $r \geq 2$ and $k \geq 2$,

$$
k^{r-1} \leq m(r, k) \leq 20 r^{2} k^{r} \ln k .
$$

Proof. Suppose that an $r$-uniform hypergraph $H=(V, E)$ has less than $k^{r-1}$ edges. Consider a random coloring $f$ of $V$ with $k$ colors such that every vertex gets colored with color $i$ with probability $1 / k$ for every $1 \leq$ $i \leq k$ independently of all other vertices. Then for every edge $e \in E$, the probability that $e$ is monochromatic is $k^{1-r}$ and the expected number of monochromatic edges is $|E| k^{1-r}<1$. Thus there exists a $k$-coloring of $V$ with no monochromatic edges.

To prove the upper bound, let $m=\left\lfloor 20 r^{2} k^{r} \ln k\right\rfloor$. If $m \geq\left(\begin{array}{c}1+k r \\ r\end{array}\right)$, then the complete hypergraph $K_{1+k r}^{r}$ witnesses the bound, so we assume the opposite. Consider a random hypergraph $G(r, k, m)$ on a set $V$ of $k r^{2}$ labelled vertices, where every of $\left(\begin{array}{c}k r^{2} \\ r\end{array}\right) r$-subsets of $V$ belongs to $E(G(r, k, m))$ with probability $p=0.5 m\left(\begin{array}{c}k r^{2} \\ r\end{array}\right)$ independently of all other $r$-subsets. Note that

$$
\operatorname{Pr}\{|E(G(r, k, m))|>m\}<1 / 2 .
$$

For a given $W \subset V$ with $|W|=r^{2}$, the probability that $W$ is independent is at most

$$
(1-p)^{\left(\begin{array}{c}
r^{2} \\
r
\end{array}\right)} \leq \exp \left\{-\left(\begin{array}{c}
r^{2} \\
r
\end{array}\right) \cdot 0.5 m\left(\begin{array}{c}
k r^{2} \\
r
\end{array}\right)^{-1}\right\} \leq \exp \left\{-\frac{0.5 m}{e k^{r}}\right\} .
$$


Therefore, since $m>19.5 r^{2} k^{r} \ln k$, the probability that there is some independent $W \subset V$ with $|W|=r^{2}$ is at most

$$
\begin{aligned}
\left(\begin{array}{c}
k r^{2} \\
r^{2}
\end{array}\right) \exp \left\{-\frac{m}{2 e k^{r}}\right\} & \leq(e k)^{r^{2}} \exp \left\{-\frac{19.5 r^{2} \ln k}{2 e}\right\} \\
& \leq \exp \left\{r^{2}(1+\ln k)-3.5 r^{2} \ln k\right\} .
\end{aligned}
$$

The last expression is at most $\exp \left\{-0.5 r^{2}\right\}<1 / 4$, and with positive probability $G(r, k, m)$ has at most $m$ edges and has no independent set of size $r^{2}$, which means that it is not $k$-colorable. This proves the lemma.

Remark 2. The proof of the lower bound works for list colorings as well.

Beck [8] improved the lower bound for $m(r, 2)$ to $2^{r} r^{1 / 3-\varepsilon}$ and Spencer [48] presented a simpler proof of the Beck's bound based on random recoloring. Recently, Radhakrishnan and Srinivasan [44] improved the lower bound further.

Theorem 17 [44]. For every $c<1 / \sqrt{2}$, there exists an $r_{0}=r_{0}(c)$ such that

$$
m(r, 2) \geq c 2^{r} \sqrt{r / \ln r}
$$

for every $r>r_{0}$.

Remark 3. In fact, the proof of Theorem 17 also can be adapted for list coloring. So, the result holds for $L$-critical $r$-uniform hypergraphs for every 2-uniform list $L$.

Erdős [16] and Erdős and Lovász [18] said that "perhaps, the order of magnitude of $m(r, 2)$ is $r 2^{r}$ ". The following result supports the insight of Erdős.

Theorem 18 [25]. For every positive integer $k$, let $c=c(k)=\exp \left\{-4 k^{2}\right\}$ and $r_{k}=\exp \left\{2 c_{k}^{-2}\right\}$. Let $n$ be a positive integer such that $k \geq 2^{n}$. Then for every $r>r_{k}$,

$$
m(r, k) \geq c k^{r}\left(\frac{r}{\ln r}\right)^{\frac{n}{n+1}} .
$$

Note that the proof of Theorem 18 does not work for list coloring. Mubayi and Tetali [41] have some other results for fixed $k$ and large $r$.

Recall that the ratio of the RHS of (14) to the LHS is $20 r^{2} k \ln k$. When $k$ is larger than $r$, then the factor $k \ln k$ becomes more important than $r^{2}$. Alon [4] improved both bounds in (14) for $k$ large in comparison with $r$. 
Theorem 19 [4]. For every positive integers $r \geq 3$ and $k \geq 2$,

$$
m(r, k) \leq\left(\begin{array}{c}
k(r-1)+1 \\
r
\end{array}\right) \frac{\ln r}{\ln r-1}\left\lfloor\frac{r}{\ln r}\right\rfloor^{-1}<\left(\begin{array}{c}
k(r-1)+1 \\
r
\end{array}\right)
$$

and

$$
m(r, k)>(r-1)\left\lceil\frac{k}{r}\right\rceil\left\lfloor\frac{r-1}{r}\right\rfloor^{r-1} \sim \frac{1}{e} k^{r} .
$$

Furthermore, if $r \rightarrow \infty$ and $k / r \rightarrow \infty$, then

$$
m(r, k)=O\left(\left(\begin{array}{c}
k(r-1)+1 \\
r
\end{array}\right) r^{1.5} \ln r\left(\frac{3}{4}\right)^{r}\right)
$$

Note that when $k$ is much larger than $r$, the complete hypergraph $K_{1+k(r-1)}^{r}$ gives a better upper bound than (14), but Alon's bound is even better. The proof of the lower bound is amazingly simple: he first colors vertices of a hypergraph at random using most of the colors, but not all. Then he uncolors a vertex in every monochromatic edge and spends a new color for every $r-1$ uncolored vertices. This proof does not work for list coloring; thus it would be interesting to find a reasonable lower bound for the number of edges in $L$-critical $r$-uniform hypergraphs for arbitrary $k$ uniform lists $L$. Also, with respect to $k$, the upper and lower bounds are of the same order, but with respect to $r$, the gap probably could be narrowed.

If a hypergraph $H=(V, E)$ is not uniform but $\sum_{e \in E} 2^{-|e|} \leq 1 / 2$, then a random 2-coloring (as in the proof of Lemma 16) with positive probability is proper. Erdős and Lovász [18] conjectured that the minimum value $\phi(n)$ of $\sum_{e \in E} 2^{-|e|}$ over non-2-colorable hypergraphs with the minimum size of an edge equal to $n$ tends to infinity as $n$ tends to infinity. Beck [8] proved this conjecture. The lower bound on $\phi(n)$ in his proof tends to infinity rather slowly. It would be interesting to estimate the rate of growth of $\phi(n)$.

\section{ON CRITICAL SIMPLE HYPERGRAPHS}

A hypergraph is called simple (sometimes, linear) if no two distinct edges share more than one vertex. Let $m^{*}(r, k)$ denote the minimum number of edges in an $r$-uniform not $k$-colorable simple hypergraph. Since Fano plane is a simple hypergraph, $m^{*}(3,2)=m(3,2)=7$. But in general, $m^{*}(r, k)$ grows much faster than $m(r, k)$. In their seminal paper [18], Erdős and 
Lovász thoroughly studied $m^{*}(r, k)$. In fact, the celebrated Local Lemma appeared in this paper and its first application was to give lower bounds on $m^{*}(r, k)$.

Theorem 20 [18]. Let $s \geq 2, r \geq 2, k \geq 2, n=4 \cdot 20^{s-1} r^{3 s-2} k^{(s-1)(r+1)}$, $m=4 \cdot 20^{s} r^{3 s-2} k^{s(r+1)}, d=20 r^{2} k^{r-1}$. Then there exists an $r$-uniform hypergraph $H$ on $k n$ vertices with at most $m$ edges and with degrees at most $d$ which does not contain any circuit of length $\leq s$ and in which each set of $n$ vertices contains an edge. In particular, $H$ is not $k$-colorable.

Since for a hypergraph being simple is the same as to have no 2-circuits, pluging in $s=2$ yields

$$
m^{*}(r, k) \leq 1600 r^{2} k^{2(r+1)},
$$

and this is still the best known bound for $r$ large in comparison with $k$.

Theorem 21 [18]. Let $r \geq 2, k \geq 2$. Then

$$
m^{*}(r, k) \geq \frac{k^{2(r-2)}}{16 r(r-1)^{2}} .
$$

This bound can be improved by a factor of $r / 2$ as follows. Theorem 5 in [18] says that every simple $(k+1)$-chromatic $r$-uniform hypergraph contains at least $k^{r-2} / 4(r-1)$ vertices with degree at least $k^{r-2} / 4(r-1)$. Then simply the sum of degrees of vertices is used. But one can be less generous. Let $G$ be a $(k+1)$-chromatic $r$-uniform hypergraph. Order the vertices $v_{1}, v_{2}, \ldots$ of $G$ so that $\operatorname{deg}_{G}\left(v_{1}\right) \geq \operatorname{deg}_{G}\left(v_{2}\right) \geq \ldots$ and delete one by one vertices in this order together with the incident edges. The degree of a vertex $v_{i}$ at the moment of deletion is at least $\operatorname{deg}_{G}\left(v_{i}\right)-(i-1)$, because $G$ is simple. Thus by the cited above Theorem 5 in [18], after deleting vertex $v_{k^{r-2} / 4(r-1)}$ we have deleted at least

$$
\frac{k^{r-2}}{4(r-1)}+\left(\frac{k^{r-2}}{4(r-1)}-1\right)+\left(\frac{k^{r-2}}{4(r-1)}-2\right)+\cdots+1 \geq \frac{k^{2 r-4}}{32(r-1)^{2}}
$$

edges. This proves the bound.

For $k=2$, the lower bound can be improved further. Szabó [50] proved that for every $\varepsilon>0$ there exists $r_{0}(\varepsilon)$ such that for $r \geq r_{0}(\varepsilon)$ every 3chromatic $r$-uniform simple hypergraph has a vertex of degree at least $2^{r} r^{-\varepsilon}$. Using this result one gets along the lines of the proof of Theorem 5 in [18] 
and of the previous paragraph that for every $\varepsilon>0$ there exists $r_{0}(\varepsilon)$ such that

$$
m^{*}(r, 2) \geq \frac{k^{2 r-2}}{2(r-1)^{2 \varepsilon}}
$$

for $r \geq r_{0}(\varepsilon)$.

For $k$ very large in comparison with $r$, the bounds on $m^{*}(r, k)$ were improved and generalized to partial $(r, l)$-systems. A partial $(r, l)$-system is an $r$-uniform hypergraph in which every set of $l$ vertices is contained in at most one edge. Let $m(r, k, l)$ be the minimum number of edges in an $(r, l)$ system that is not $k$-colorable. Thus, a simple $r$-uniform hypergraph is a partial $(r, 2)$-system and $m^{*}(r, k)=m(r, k, 2)$.

The works $[43,45,22]$ on Steiner systems with small independence number yield results for partial $(r, l)$-systems, and imply upper bounds on $m^{*}(r, k)$ that improve (15) for $k$ very large in comparison with $r$. In particular, Grable, Phelps and Rödl [22] constructed simple hypergraphs (in fact, Steiner systems) with chromatic number at least $k+1$ and at most $c 4^{r} r^{2} k^{2 r-2} \ln ^{2} k$ edges for every $r$ and infinitely many $k$. Thus, for such $r$ and $k$,

$$
m^{*}(r, k) \leq c 4^{r} r^{2} k^{2 r-2} \ln ^{2} k
$$

Kostochka, Mubayi, Rödl, and Tetali [27] proved that for every $r \geq 3$, $l \geq 2$,

$$
m(r, k, l) \leq \frac{\left(2 r^{l}\right)^{\frac{3 l}{l-1}}}{r(r-1) \ldots(r-l+1)}\left(k^{r-1} \ln 3 k\right)^{\frac{l}{l-1}} .
$$

For fixed $r$ and huge $k$, this bound was matched by the following lower bound.

Theorem 22 [27]. Let $r>l \geq 2$ be fixed. Then there exists $C$ depending only on $r$ and $l$ such that

$$
m(r, k, l) \geq C\left(k^{r-1} \ln k\right)^{l /(l-1)} .
$$

The proof of Theorem 22 does not work for list coloring. 


\section{VARIATIONS: PANCHROMATIC AND STRONG COLORINGS}

One of reasonable generalizations of hypergraph coloring is the panchromatic $k$-coloring - a $k$-coloring such that every edge meets every of $k$ colors. Then the ordinary 2-coloring is a panchromatic 2-coloring. Let $p(r, k)$ denote the minimum number of edges in an $r$-uniform hypergraph not admitting any panchromatic $k$-coloring. By above, $p(r, 2)=m(r, 2)$.

Theorem 3 in the already mentioned paper [18] by Erdős and Lovász speaks on panchromatic colorings.

Theorem 23 [18]. If each edge of an $r$-uniform hypergraph $H$ meets at most $k^{r-1} / 4(k-1)^{r}$ other edges, then $H$ is panchromatically $k$-colorable.

This implies that

$$
p(r, k)>1+\frac{k^{r-1}}{4(k-1)^{r}}>1+\frac{1}{4 k} e^{r / k} .
$$

Let $N(k, r)$ denote the minimum number of vertices in a $k$-partite graph with list chromatic number greater than $r$. Among other results, Erdős, Rubin, and Taylor [19] proved that $N(2, r)$ is closely connected with $m(r, 2)$ :

$$
m(r, 2) \leq N(2, r) \leq 2 m(r, 2)
$$

An interesting feature of this results is that ORDINARY coloring of $r$-uniform hypergraphs relates to LIST coloring of bipartite graphs. This relation can be easily extended to panchromatic colorings with more colors:

Theorem $24[26]$. For every $r \geq 2$ and $k \geq 2, p(r, k) \leq N(k, r) \leq k p(r, k)$.

It follows from Alon's results in [5] that for some $0<c_{1}<c_{2}$ and every $r \geq 2$ and $k \geq 2$,

$$
\exp \left\{c_{1} r / k\right\} \leq N(k, r) \leq k \exp \left\{c_{2} r / k\right\} .
$$

Therefore, by Theorem 4 we get reasonable bounds on $p(r, k)$ for fixed $k$ and large $r$ :

$$
\exp \left\{c_{1} r / k\right\} / k \leq p(r, k) \leq k \exp \left\{c_{2} r / k\right\} .
$$

Recall that Theorem 23 also yields the lower bound on $p(r, k)$ with $c_{1}=1 / 4$ and thus itself implies the lower bound $1+\frac{1}{4 k} e^{r / k}$ on $N(k, r)$. 
One can also define panchromatic list colorings: If each vertex $v$ of $H$ is assigned a list $L(v)$ of $k$ colors, then a panchromatic $L$-coloring of $H$ is a coloring in which each vertex is given a color from its own list and each edge contains vertices with at least $k$ different colours.

Kostochka and Woodall [33] obtained bounds on the minimum number of edges in hypergraphs being edge critical with respect to panchromatic colorings.

Theorem 25 [33]. Let $k \geq 2$ and let $H=(V, E)$ be a hypergraph in which every edge has at least $k$ vertices, and every vertex is given a list $L(v)$ of $k$ colors. If $H$ is not panchromatically $L$-colorable, but after deleting any edge becomes panchromatically $L$-colorable, then $|E| \geq(|V|+k-2) /(k-1)$. This bound is attained for every $k \geq 2$ for ordinary panchromatic colorings.

If the condition 'every edge has at least $k$ vertices' is replaced with ' $k$ uniform', then the inequality can be strengthened.

Theorem 26 [33]. Let $k \geq 2$ and let $H=(V, E)$ be a $k$-uniform hypergraph and every vertex is given a list $L(v)$ of $k$ colors. If $H$ is not panchromatically $L$-colorable, but after deleting any edge becomes panchromatically $L$-colorable, then $|E| \geq|V|(k+2) / k^{2}$.

This bound is unlikely to be sharp. The following result says that even if panchromatically critical hypergraphs are not dense themselves, they must contain dense subgraphs.

Theorem 27 [33]. Let $k \geq 4, k \neq 5$, and let $H=(V, E)$ be a $k$-uniform hypergraph such that

$$
\left|E\left(H\left(V^{\prime}\right)\right)\right| \leq \frac{k\left|V^{\prime}\right|-k+1}{k^{2}-2 k+2}, \quad \text { whenever } \quad V^{\prime} \subseteq V, \quad E\left(H\left(V^{\prime}\right)\right) \neq \emptyset .
$$

Then $H$ is panchromatically $k$-colorable. For $k \in\{3,5\}$, the same conclusion follows if the final +1 in the numerator is omitted.

This bound is sharp if $k \notin\{3,5\}$. Note that a panchromatic $k$-coloring of a $k$-uniform hypergraph is a strong coloring, i.e. the coloring in which every two distinct vertices sharing an edge must have different colors. Every strong coloring of a hypergraph $H$ corresponds to a proper edge coloring of the hypergraph $H^{*}$ dual to $H$. The problem of estimating the edge chromatic number of uniform hypergraphs with a given maximum degree and moderate codegree attracted a lot of attention after Rödl's solution 
of the Erdős-Hanani Problem. A remarkable sequence of significant papers due to Rödl, Frankl, Pippenger, Spencer, Kahn, Grable, Alon, Kim, Molloy, Reed, and $\mathrm{Vu}$ was devoted to this topic. Theorem 27 can be interpreted as a (somewhat unusual) sufficient condition for the edge-chromatic number of a hypergraph $H$ to equal its trivial lower bound, the maximum vertex degree $\Delta(H)$.

Theorem 28 [33]. Let $H$ be a hypergraph with maximum degree $r$, where $r=4$ or $r \geq 6$. If every vertex subset $S$ is incident with at least $\left(\left(r^{2}-2 r+2\right)|S|+r-1\right) / r$ edges, then $H$ is $r$-edge-colorable.

By the definition, the strong chromatic number of a hypergraph $H=$ $(V, E)$ equals the chromatic number of its skeleton, $S(H)$ - the graph on $V$ whose vertices are adjacent if and only if they share some edge in $H$. Deletion of an edge from a hypergraph is a rather rough action with respect to strong coloring: deletion of an edge of size $r$ may reduce the strong chromatic number by $r-1$. A subtler operation is splitting: if $H=(V, E)$ is a hypergraph, $v \in e \in E$, and $\operatorname{deg}_{H}(v) \geq 2$, then the $(v, e)$-splitting of $H$ is obtained by replacing the edge $e$ by the edge $e-v+v^{\prime}$, where $v^{\prime}$ is a new vertex. Then deleting an edge $e$ can be performed as a sequence of $(v, e)$ splittings over the vertices $v \in e$ of degree at least two. The $(v, e)$-splitting corresponds to cutting edge $v$ in the dual hypergraph $H^{*}$ into two pieces, one of which has size one.

Kostochka and Woodall [34] considered splitting-critical hypergraphs with respect to strong coloring and strong list-coloring. It appears that for $k \geq r+2$, the sparsest $k$-splitting-critical $r$-uniform hypergraphs are obtained from sparse $k$-critical graphs by adding to every edge $r-2$ new vertices (of degree one in the resulting hypergraph). On the other hand, the sparsest $(r+1)$-splitting-critical $r$-uniform hypergraphs cannot be obtained this way. If $k$ is large in comparison with $r$ and the skeleton $S(H)$ of a $k$-splitting-critical $r$-uniform hypergraph $H$ has no large cliques, then the lower bound on the number of edges in $H$ can be improved.

Theorem 29 [34]. Let $s \geq r$ be positive integers and let $k$ be sufficiently large with respect to $s$. Let $H$ be a list- $k$-splitting-critical $r$-uniform hypergraph with respect to strong coloring whose skeleton $S(H)$ does not contain a complete subgraph on $s+1$ vertices. Then

$$
|E(H)| \geq k\left(1-6(\ln k)^{-1 / 3}\right)(|V(H)|-(r-2)|E(H)|) .
$$


As with Theorem 13, the bad side of the last theorem is that the proof works only for really large $k$, and the good side of it is that the bound is asymptotically (in $k$ ) sharp even for hypergraphs of large girth.

\section{Concluding Remarks}

Certainly, the survey is not full. Essentially, it describes problems I am interested in. The reader might look into [23, Chapter 5] and [46] for more problems on color-critical graphs and hypergraphs. Maybe some proofs of the results above can be simplified using recent impressive results of $\mathrm{Vu}$ (see, e.g., $[55])$.

I thank Michael Stiebitz and Douglas Woodall for our discussions on the topic and their helpful comments on a earlier version of this survey. Some pieces of our joint works were used in this text. Thanks for helpful comments are also due to Oleg Borodin and Bjarne Toft.

\section{REFERENCES}

[1] H. L. Abbott and D. R. Hare, Sparse color-critical hypergraphs, Combinatorica, 9 (1989), 233-243.

[2] H. L. Abbott, D. R. Hare and B. Zhou, Sparse color-critical graphs and hypergraphs with no short cycles, J. Graph Theory, 18 (1994), 373-388.

[3] H. L. Abbott, D. R. Hare and B. Zhou, Color-critical graphs and hypergraphs with few edges and no short cycles, Discrete Math., 182 (1998), 3-11.

[4] N. Alon, Hypergraphs with high chromatic number, Graphs and Combinatorics, 1 (1985), 387-389.

[5] N. Alon, Choice number of graphs: a probabilistic approach, Combinatorics, Probability and Computing, 1 (1992), 107-114.

[6] N. Alon, Restricted colorings of graphs, in: K. Walker, ed., "Surveys in Combinatorics, 1993", London Math. Soc. Lecture Note Series, 187 (Cambridge Univ. Press, Cambridge, UK, 1993), 1-33.

[7] D. Archdeacon, J. Hutchinson, A. Nakamoto, S. Negami and K. Ota, Chromatic numbers of quadrangulations on closed surfaces, J. Graph Theory 37 (2001), 100114.

[8] J. Beck, On 3-chromatic hypergraphs, Discrete Math., 24 (1978), 127-137. 
[9] M. I. Burstein, Critical hypergraphs with minimal number of edges (Russian), Bull. Acad. Sci. Georgian SSR, 83 (1976), 285-288.

[10] W. A. Deuber, A. V. Kostochka and H. Sachs, A shorter proof of Dirac's theorem on the number of edges in chromatically critical graphs, Diskretnyi Analiz $i$ Issledovanie Operacii, 3 (1996), No. 4, 28-34 (in Russian).

[11] G. A. Dirac, Note on the colouring of graphs, Math. Z., 54 (1951), 347-353.

[12] G. A. Dirac, The structure of k-chromatic graphs, Fund. Math., 40 (1953), 42-55.

[13] G. A. Dirac, A theorem of R. L. Brooks and a conjecture of H. Hadwiger, Proc. London Math. Soc., (3) 7 (1957), 161-195.

[14] G. A. Dirac, The number of edges in critical graphs, J. Reine u. Angew. Math., 268/269 (1974), 150-164.

[15] P. Erdős, On a combinatorial problem, I, Nordisk Mat. Tidskrift, 11 (1963), 5-10.

[16] P. Erdős, On a combinatorial problem, II, Acta Mathematica of the Academy of Sciences, Hungary, 15 (1964), 445-447.

[17] P. Erdős and A. Hajnal, On a property of families of sets, Acta Mathematica of the Academy of Sciences, Hungary, 12 (1961), 87-123.

[18] P. Erdős and L. Lovász, Problems and Results on 3-chromatic hypergraphs and some related questions, in: Infinite and Finite Sets, A. Hajnal et. al., editors, Colloq. Math. Soc. J. Bolyai, 11, North Holland, Amsterdam, 609-627, 1975.

[19] P. Erdős, A. L. Rubin and H. Taylor, Choosability in graphs, in: Proc. West Coast Conference on Combinatorics, Graph Theory and Computing, Arcata, 1979, Congr. Numer., 26 (1980), 125-157.

[20] T. Gallai, Kritische Graphen I, Publ. Math. Inst. Hungar. Acad. Sci., 8 (1963), $165-192$.

[21] T. Gallai, Kritische Graphen II, Publ. Math. Inst. Hungar. Acad. Sci., 8 (1963), 373-395.

[22] D. Grable, K. Phelps and V Rödl, The minimum independence number for designs, Combinatorica, 15 (1995), 175-185.

[23] T. R. Jensen and B. Toft, Graph coloring problems, Wiley-Interscience, 1995.

[24] A. V. Kostochka, Constructing strictly $k$-degenerate $k$-chromatic graphs of arbitrary girth, Abstracts of the V All-Union Conference on the Problems of Theoretical Cybernetics, Novosibirsk, 1980, 130-131 (in Russian).

[25] A. V. Kostochka, Coloring uniform hypergraphs with few colors, submitted.

[26] A. V. Kostochka, On a theorem by Erdős, Rubin and Taylor, submitted.

[27] A. V. Kostochka, D. Mubayi, V. Rödl and P. Tetali, On the chromatic number of set-systems, Random Structures and Algorithms, 19 (2001), 87-98.

[28] A. V. Kostochka and J. Nešetřil, Properties of Descartes' construction of trianglefree graphs with high chromatic number, Combinatorics, Probability and Computing, 8 (1999), 467-472. 
[29] A. V. Kostochka and M. Stiebitz, Excess in colour-critical graphs, Bolyai Society Mathematical Studies, 7 (1999), 87-99.

[30] A. V. Kostochka and M. Stiebitz, On the number of edges in colour-critical graphs and hypergraphs, Combinatorica, 20 (2000), 521-530.

[31] A. V. Kostochka and M. Stiebitz, A list version of Dirac's theorem on the number of edges in colour-critical graphs, Journal of Graph Theory, 39 (2002), 165-167.

[32] A. V. Kostochka and M. Stiebitz, A new lower bound on the number of edges in colour-critical graphs, to appear in J. Combinatorial Theory B.

[33] A. V. Kostochka and D. R. Woodall, Density conditions for panchromatic colourings of hypergraphs, Combinatorica, 21 (2001), 515-541.

[34] A. V. Kostochka and D. R. Woodall, On the number of edges in hypergraphs critical with respect to strong colourings, European Journal of Combinatorics, 21 (2000), 249-255.

[35] M. Krivelevich, An improved bound on the minimal number of edges in colorcritical graphs, Electron J. Combin., 5 (1998), no. 1, Research Paper 4, 4 pp.

[36] M. Krivelevich, On the minimal number of edges in color-critical graphs, Combinatorica, 17 (1997), 401-426.

[37] H. V. Kronk and J. Mitchem, On Dirac's generalization of Brooks' theorem, Canad. J. Math., 24 (1972), 805-807.

[38] L. Lovász, A generalization of Konig's theorem, Acta Math. Acad. Sci. Hungar., 21 (1970), 443-446.

[39] L. Lovász, Coverings and colorings of hypergraphs, in: Congressus Numer., 8 (1973), 3-12.

[40] J. Mitchem, A new proof of a theorem of Dirac on the number of edges in critical graphs, J. Reine u. Angew. Math., 299/300 (1978), 84-91.

[41] D. Mubayi and P. Tetali, Generalizing Property B to many colors, manuscript.

[42] O. Ore, The Four Colour Problem, Academic Press, New York, 1967.

[43] K. Phelps, V Rödl, Steiner Triple Systems with Minimum Independence Number, Ars combinatoria, 21 (1986), 167-172.

[44] J. Radhakrishnan and A. Srinivasan, Improved bounds and algorithms for hypergraph two-coloring, Random Structures and Algorithms, 16 (2000), 4-32.

[45] V. Rödl, E. Šinajová, Note on Independent Sets in Steiner Systems, Random Structures and Algorithms, 5 (1994), 183-190.

[46] H. Sachs and M. Stiebitz, On constructive methods in the theory of colour-critical graphs, Discrete Math., 74 (1989), 201-226.

[47] P. D. Seymour, On the two-coloring of hypergraphs, Quart. J. Math. Oxford, 25 (1974), 303-312.

[48] J. Spencer, Coloring $n$-sets red and blue, J. Comb. Theory Ser. A, 30 (1981), $112-113$. 
[49] M. Stiebitz, Proof of a conjecture of T. Gallai concerning connectivity properties of colour-critical graphs, Combinatorica, 2 (1982), 315-323.

[50] Szabó, An application of Lovász' Local Lemma - a new lower bound for the van der Waerden number, Random Structures and Algorithms, 1 (1990), 344-360.

[51] B. Toft, Colour-critical graphs and hypergraphs, J. Combin. Th. Ser. B, 16 (1974), $145-161$.

[52] B. Toft, Colouring, stable sets and perfect graphs, Graham, R. L. (ed.) et al., Handbook of combinatorics. Vol. 1-2. Amsterdam: Elsevier (North-Holland), 1995, $233-288$.

[53] D. R. Woodall, Property B and the four-color problem. Combinatorics. Institute of Mathematics and its Applications, Southend-on-sea, England (1972), 322-340.

[54] V. G. Vizing, Colouring the vertices of a graph with prescribed colours, Metody Diskretnogo Analiza v Teorii Kodov i Skhem, No. 29 (1976), 3-10 (in Russian).

[55] V. H. Vu, A general upper bound on the list chromatic number of locally sparse graphs, Combinatorics, Probability and Computing, 11 (2002), 103-111.

[56] J. Weinstein, Excess in critical graphs, J. Combin. Th.(B), 18 (1975), 24-31.

\author{
Alexandr Kostochka \\ University of Illinois at \\ Urbana-Champaign \\ Urbana \\ IL 61801 \\ and \\ Institute of Mathematics \\ Novosibirsk 630090 \\ Russia \\ kostochk@math.uiuc.edu
}

